\title{
Simulation Experiment on Path Planning in Incomplete Fuzzy System Based on Dominance Rough Set
}

\author{
Sheng Pu \\ School of Physical Education, Mianyang Normal University, Mianyang, 621000, China \\ sheng_pu@yeah.net
}

Keywords: Ordered information, Autonomous learning, Clustering algorithm, Rough set, Adaptability.

\begin{abstract}
In order to enhance the processing capacity of the inconsistent ordered study information system and transform the rough set of different precision dominance relation, they can enhance the adaptability of inconsistent information by introducing the clustering algorithm, because rough set can effectively deal with imprecise, inconsistent and incomplete information, it can effectively get rid of dependence on a priori knowledge in the learning process, and has strong ability of independent learning. Through the simulation analysis, we found that the autonomous learning method has higher inconsistency ordered information system with the prominent advantages. In the number of different rough set, this method has different accuracy and can be very good to adapt to the change of the offensive and defensive line as well as automatic planning prevention path, which provide the theory reference for the study of basketball training autonomous learning.
\end{abstract}

\section{Introduction}

With the growth of global economic development, competition is increasingly fierce in manufacturing industry. The core of competition has been transferred to the innovative technology and high added value of new products on the basis of competition [1,2]. In the process of new product development, in order to shorten the design cycle, improve design quality and reduce design cost, computer is introduced to the design of mechatronic simulation. Machine learning are an important field in current artificial intelligence research and the essence of machine learning is the transformation of the knowledge representation, however the data essential characteristics plays a decisive role. Because of the existence of inconsistent information in actual system, people often rely on some fields' prior knowledge in traditional machine learning, such as probability theory, fuzzy set and other research [3-5]. How to get rid of dependence on a priori knowledge of the learning process under the uncertainty condition, independent learning is a difficult problem of the artificial intelligence knowledge acquisition in the research [6]. In this paper, using the rough set algorithm designs the autonomous learning system, and self-regulated learning system is used in the basketball training, the general framework is Figure 1.

Figure 1 shows the overall design framework of rough set autonomous learning method. The use of the fuzzy semantic rules of rough set carries out the classification for data information, according to clustering algorithm we can find path planning optimization algorithm, and then we go through metadata processing tools to operate the learning database, eventually it will be applied to basketball path planning, we can get the offensive and defensive autonomous learning route. 


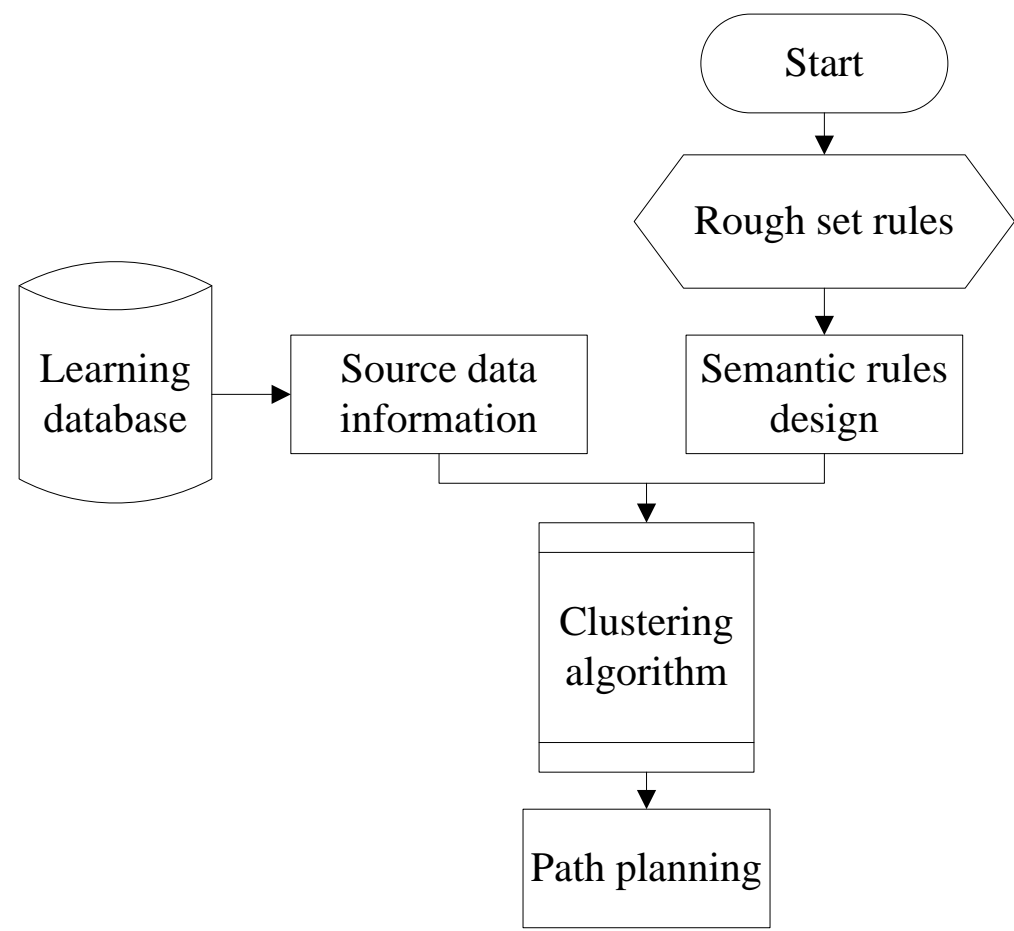

Fig.1: The overall design of autonomous learning method based on rough set

\section{Design of Basketball Autonomous Learning Dominance Relation Rough Set Algorithm}

Mechatronic modeling and simulation technology is mechanical and electrical design technology based on the intelligent network information. Compared with the traditional serial design, the design process can do collaborative work through different subsystems function by using network $[7,8]$. Because rough set can effectively deal with imprecise, inconsistent and incomplete information, it can effectively get rid of dependence on a priori knowledge in the learning process, which has the very strong self-learning ability, we can be in the fuzzy boundary value for the rough set, in which the similarity matrix can be expressed as

$$
\begin{array}{cccc}
1 & P_{12} & \cdots & P_{1 i} \\
P_{21} & 1 & \cdots & P_{2 i} \\
\vdots & \vdots & \ddots & \vdots \\
P_{j 1} & P_{j 2} & \cdots & 1
\end{array}
$$

Among them, $P_{j i}$ is the object $j$ values obtained in accordance with object $i$ certain similarity rule, if $P_{j i}$ value is larger, similarity values between two objects are smaller. Set $P \subseteq R$ and $P$ is not null, the intersection of all equivalence relations in $P$ is called an indiscernibility relationship that is denoted as:

$$
[X]_{R N D(P)}=\bigcap_{R \in P}[X]
$$

The below approximation $X$ is

$$
R^{*}(x)=\left\{x:(x \in U) \wedge\left([x]_{R} \subseteq X\right)\right\}
$$

The above approximation $X$ is

$$
R^{*}(x)=\left\{x:(x \in U) \wedge\left([x]_{R} \cap X \neq \text { emptyset }\right)\right\}
$$

The above approximate $X$ is

$$
\mu_{X}^{R}(x)=\frac{\operatorname{card}(X \cap R(x))}{\operatorname{card}(R(x))}
$$

Where $R$ is the indiscernibility relationship, it is 


$$
\mathrm{R}(\mathrm{x})=[\mathrm{x}] \mathrm{R}=\{\mathrm{y}:(\mathrm{y} \in \mathrm{U}) \wedge(\mathrm{yRx})\} .
$$

The density of data sample point $R_{k}$ can be expressed as:

$$
M_{k}=\sum_{l=1}^{n} \frac{d\left(R_{k}, R_{l}\right)}{\sum_{k=1}^{n} d\left(R_{l}, R_{e}\right)}, k=1,2, \ldots n
$$

So, we can choose a best cluster center, first of all to make the sample around density distribution is more concentrated, the density of rough set can be defined as:

$$
M \mu_{k}=\exp \left(-\frac{M_{k}}{\sum_{i=1}^{n} d\left(R_{j}, s_{i}\right)}\right), j=1,2, \ldots . n, j \neq i \text {. }
$$

In the premise of maintaining the dominance relation accuracy, we can search the best path as shown in Figure 2.

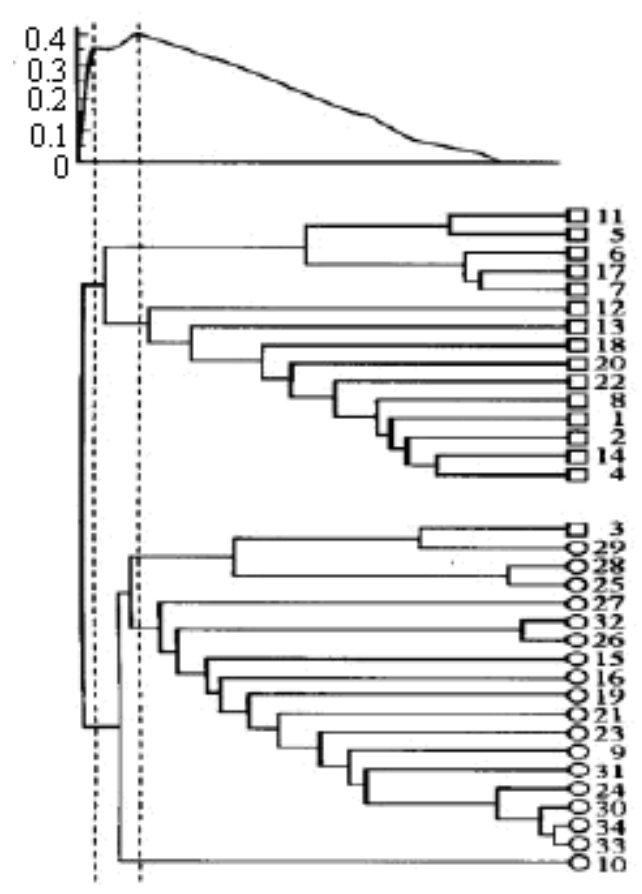

Fig.2: Search route structure diagram

Figure 2 shows the schematic diagram of the best search path in the dominance relations. Using the fuzzy boundary of rough set, we can get a series of tree structure, to carry on the path planning of each branch, finally we can obtain the optimal curves at the top, the algorithm is as follows [9]:

metamodel $1=$ attach(metadata 1$)$

metamodel2 = attach(metadata2)

tmp = metamodel 1

while(tmp != null)

If(hasDependRelationship(tmp, metamodel2))

return true

EndIf

tmp = tmp.getParent()

End While

tmp = metamodel 2

while(tmp != null)

If(hasDependRelationship(tmp, metamodel1))

return true

EndIf

tmp $=$ tmp.getParent $($ ) 


\section{The Experimental Study on the Self-access Basketball Learning Effect Simulation}

In order to verify the effectiveness and reliability of rough set self-access learning algorithm designed in second section, this paper takes the best path of attack and defense self-regulated learning in basketball training as an example [10-13]. The use of metadata management tools carries out simulation for the self-regulated basketball training learning, in which the metadata management tool interface is shown in Figure 3.

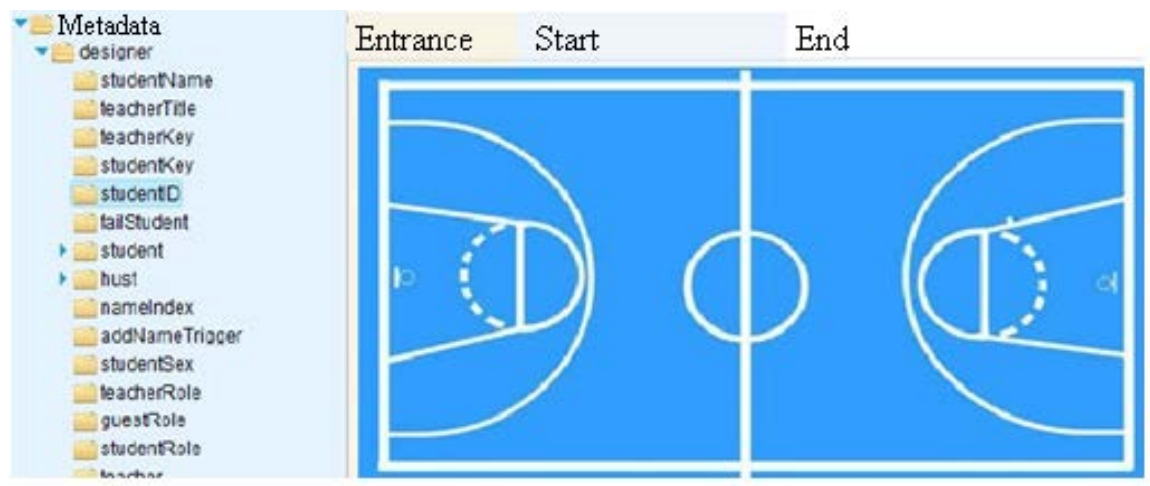

Fig.3: The metadata management tool

Metadata management tools cam improve the metadata information management. According to the need of basketball training and self-access learning, this paper sets the corresponding rough set of semantic rules to carry on semantic reasoning. Through the analysis of test results, we will get the results of clustering analysis as shown in Figure 4.

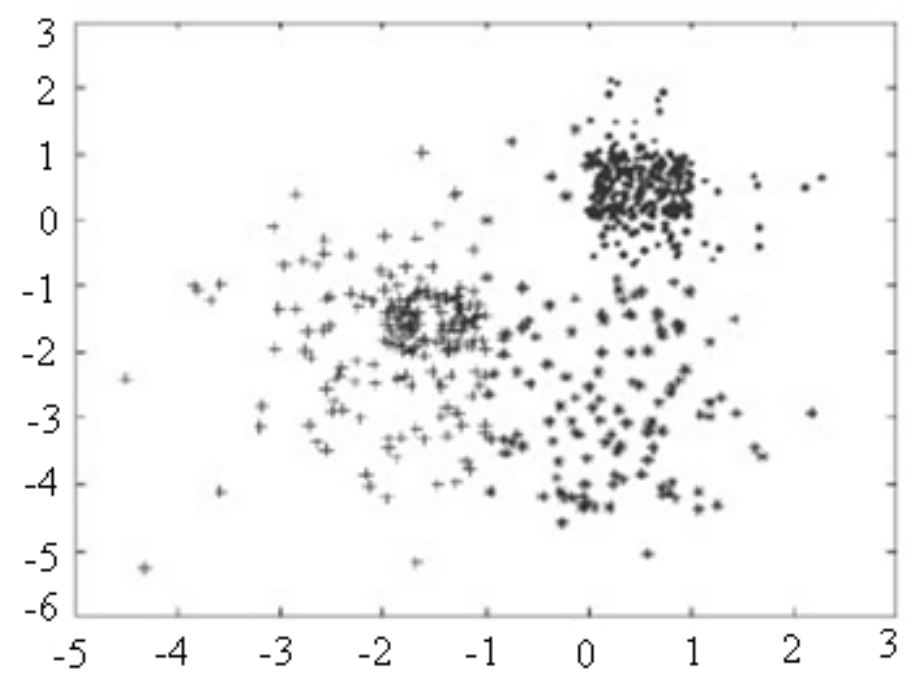

Fig.4: Clustering test results

From the clustering results, it can be seen that the algorithm has the better clustering effect on the boundary fuzzy data sample in this paper, this is because its algorithm's recombinant and non consistent mutation operation common role lead to this outcome [14]. Through the training and learning of the data set, it can better reflect the spatial organization of data samples. 


\section{Path accuracy $/ \%$}

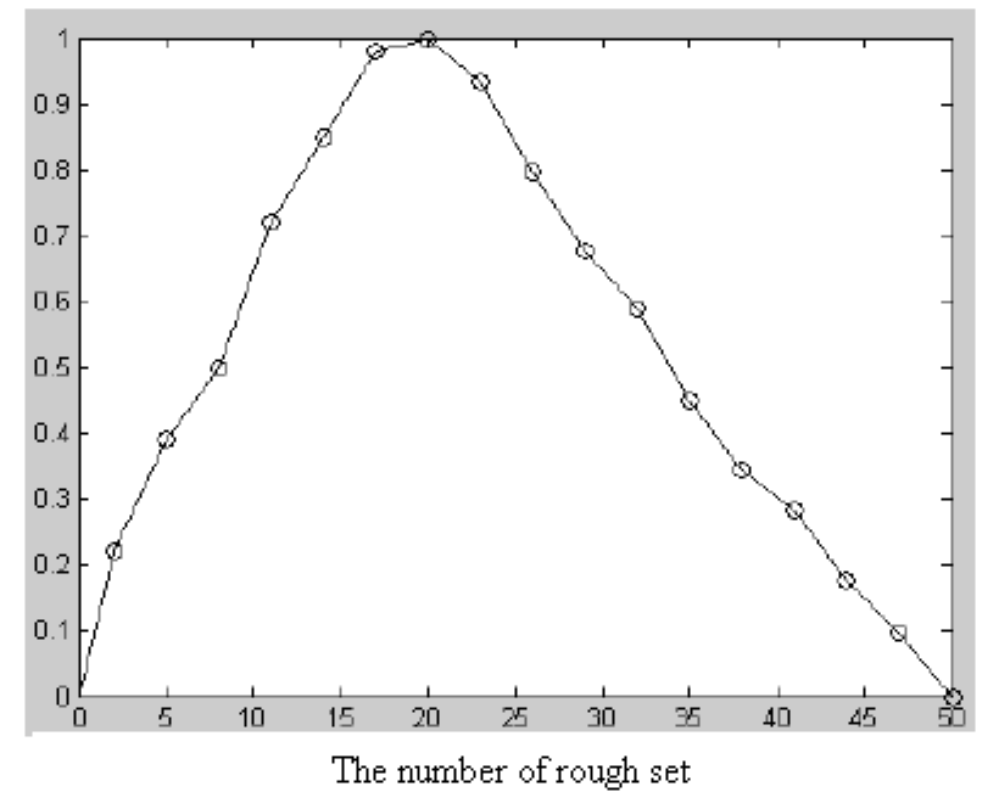

Fig.5: Path accuracy

Figure 5 shows the change curves of the path accuracy rate and the number of rough set obtained by simulation. It can be seen from the chart that along with the increase of the number of rough set, the accuracy rate is different; when the number of rough set is in 20, the accuracy rate of the path will be highest, so taking the number of rough set is 20. After the self-access learning effect simulation, we can obtain the computing results as shown in Figure 6.

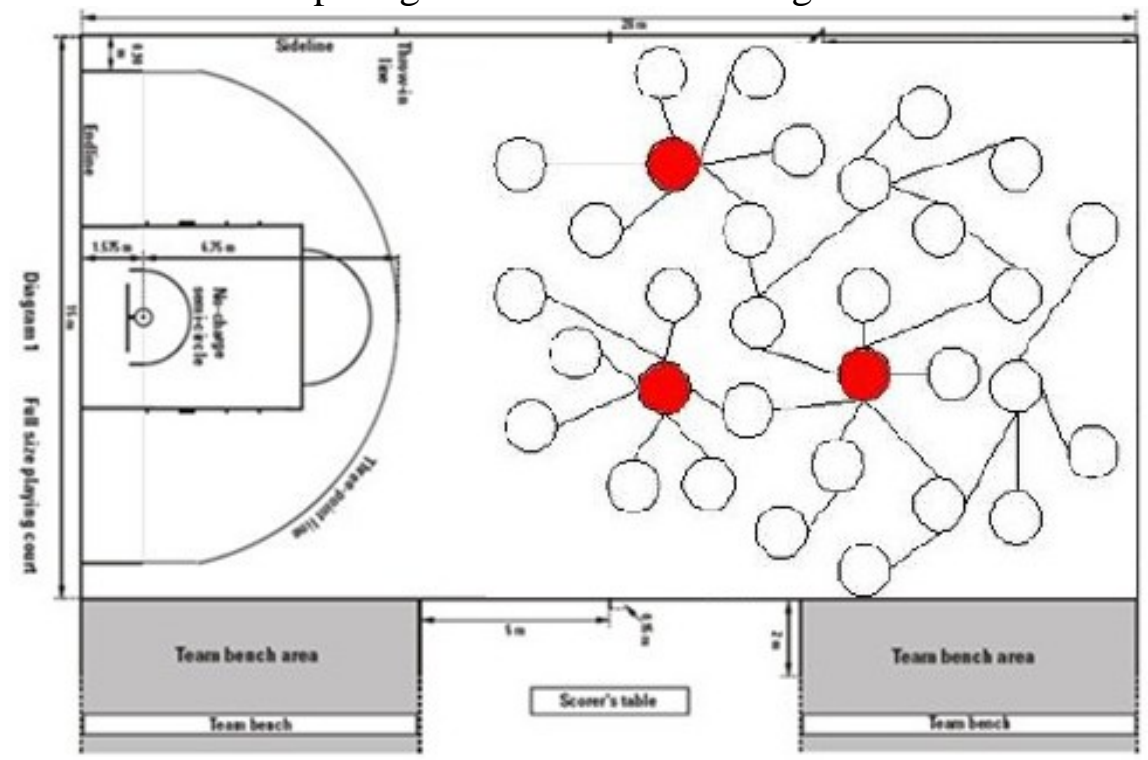

Fig.6: Basketball attack path planning results

Figure 6 shows the self-access learning structure of basketball attack and defense obtained by using the simulation. Through the machine learning of the rough set control, when meeting the front obstacles, it will automatically plan multi route, and carry out rapid calculation for each route, using the fuzzy boundary of rough set can obtain the best path [15]; when the self-access learning basketball robot gets the optimal path, it can go through independent learning to constantly adjust attack parameter, which will provide the best learning plan path for the training of basketball players.

\section{Summary}

(1) This paper designs a new kind of self-access learning and path optimization system, this system combines the advantages of rough set relationship, and using clustering algorithm carries on 
planning design for the known path, making the system has the ability of autonomous learning, and gets rid of dependence on priori knowledge in the learning process.

(2) In order to verify the effectiveness and reliability of the algorithm, using metadata processing tools carry out simulation experiment on its algorithm. Through the experiments, we found that this method has higher inconsistency ordered information system for handling and has more prominent advantages, it can obtain the adaptability of the offensive and defensive line in complex condition, which provides the theoretical reference and technical support for the basketball autonomous learning training.

\section{References}

[1] S.L. Wang, G.Y. Ding, M. Zhong. Think of spatial data mining under the large data. China Journal of thinking Electronic Research Institute, 2013(1): 78-80.

[2] B. Huang, S. Xu, W. Pu. Design and implementation of data mining platform based on MapReduce. Computer engineering and design, 2013(2): 75-76.

[3] Y. Ding, Q.P. Yang, Y.M. Qian. Research on data mining platform architecture and its key technology based on cloud computing. ZTE technology, 2013(1): 91-92.

[4] J.Y. Wang, W.X. Gu, S.H. Tan, M.H. Yin. Research on extended rules methods. Intelligent system, 2014(1): 63-64.

[5] L. Wang, G.X. Wu, J. Ji. Evidence combination rule based on neighbor thought. Computer engineering, 2010(8): 36-39.

[6] M.L. Liang, J.R. Liang, T.Z. Li. incomplete information rough set model based on $\tau$ limit tolerance relation. Computer engineering and application, 2012(31): 65-69.

[7] H. Wang, J. Wang, X. Chen. The dominance relation and knowledge discovery on double domain information system. Computer engineering and application, 2011(16): 62-63.

[8] H. Yu, S. Huang. Cutting set properties and its application of rough fuzzy sets. Journal of Luoyang Normal University, 2010(5): 101-104

[9] Q.S. Yang, G.Y. Wang, Q.H. Zhang. Interval value rough sets expansion model based on dominance relation. Journal of Shandong University, 2010(9): 95-96.

[10] W.H. Xu, X.Y. Zhang, W.X. Zhang. The below approximately reduction of inconsistent information systems under the dominance relation. Computer engineering, 2011(18): 42-43.

[11] S.B. Sun, Q.T. Wu. The rough fuzzy rough set mode knowledge based on generalized variable precision. Computer science, 2012(9): 56-57.

[12] X.C. Gui, H. Peng. Study of distribution reduction and maximum distribution reduction problem under the dominance relations. Computer engineering and applications, 2011(2):31-32.

[13] R.S. Cheng, D.Q. Miao, Y. Liu. A new approximation operator of rough fuzzy sets. Journal of Tongji University, 2011(6): 52-53.

[14] W.H. Xu, W.X. Zhang. Distribution of inconsistent information systems based on dominance relation. Fuzzy system and math, 2011(4): 102-104.

[15] Y. Liu, N. Jing, L. Chen. K-nearest neighbor connect algorithm under the MapReduce framework based on R- tree. Journal of software, 2013(8): 136-137. 